\title{
Aspectos psicosociales y éticos del dopaje
}

Psychosocial and ethical aspects of doping

Rubén Alberto Cadavid Villa, M. D., M. G., Ph.D.:

\section{Resumen}

Palabras clave: Cultura, deporte, vida, lógica y pensamiento humano, libertad, conducta social, juego limpio, ética en el deporte, deportividad, lo psicológico, dopaje.

La cultura, con su conjunto de rasgos espirituales, materiales y afectivos, caracteriza a la sociedad o al grupo. En ella se engloban modos de vida, sistemas de valores y derechos fundamentales del ser humano, tradiciones y creencias. En este contexto emergen el deporte y la competencia sana, los cuales, pese a la influencia de factores económicos, sociales y políticos, tienen la capacidad de afectar la actitud de la comunidad, fortalecer la convivencia, el desarrollo, y garantizar el proceso de formación de la personalidad de los individuos, los valores y el desarrollo integral del ser humano y la sociedad.

Se gesta el juego, que es libertad sana, emoción, fantasía y disciplina al mismo tiempo. Con el juego, la práctica del mismo y la competencia, tan tradicional y tan antigua como el ser humano, toman cuerpo, con su carácter lúdico, de cohesión social, de emulación de calidades físicas y técnicas con observación a reglas, valores y ética, para nutrir la misma sociedad. Sin embargo, la práctica del deporte y la competición sana exigen juego limpio, observación de los principios que imponen el derecho a la vida, a la salud integral, a su libertad. Por tanto, la competencia en el deporte es ajena a prácticas nocivas, como el dopaje, que envilecen los valores más sagrados del deporte: la ética y el juego limpio.

La construcción de un mundo mejor, más pacífico, más auténtico, que proteja principalmente a la juventud, se logrará si unidos todos los estamentos de la sociedad y del deporte, en su conjunto, luchan para detener el tráfico y el consumo de las drogas de uso ilícito, la práctica del dopaje, porque el deporte debe estar al servicio del desarrollo armonioso de la sociedad, y de su principal razón de ser: el ser humano.

\section{Abstract}

Culture is a set of spiritual, material and affective features that shape a society or group. It encompasses lifestyles, value systems, fundamental human rights, traditions and beliefs. This is the context for the rising of sports and healthy competition, which, despite the influence of economic, social and political factors, have the potential to affect a community's attitude, strengthen coexistence and development, and guarantee the individuals' personal formation process, and values as well as the integral development of human beings and society as a whole.

Play comes to life as a simultaneous expression of healthy freedom, emotion, fantasy and discipline. Play, along with its practice and competition -as traditional and old as the human being itself- arise with their ludic character, marked by social cohesiveness, emulation of physical and technical qualities following the rules, ethics and values, to nourish the same society. The practice of sport and healthy competition calls for fair play, respect for the principles that impose the right to life, integral health, and freedom. Therefore, sport competition has nothing to do with improper practices like doping, which degrade the most sacred sport values: ethics and fair play.

Building a better, more peaceful and authentic world, that protects especially the youngsters, only will be possible if all the bodies of society and sports, working together as one strive for stopping drug dealing and consumption, doping, because sports must be at the service of the harmonious development of society and its raison detre: the human being.

Key words: $\quad$ Culture, sport, life, logics and human thought, freedom, social behavior, fair play, ethics of sports, sportsmanship, psychological issues, doping.

Fecha de recepción: 15 de noviembre de 2005

Fecha de aceptación: 11 de agosto de 2006

* Director Programa Profesional de Cultura Física y Deporte, Universidad Incca de Colombia, Bogotá. Profesional especializado, Hospital Militar Central, Bogotá. Médico especializado en Medicina del Deporte, Cardiología y Fisiología del Ejercicio. Profesor Universitario e investigador en Ciencias del deporte. Presidente de la Comisión Médica Deportiva de la División Mayor del Fútbol Colombiano, Dimayor, y de la Federación Colombiana de Ciclismo (Programa de Control al Dopaje). Coordinador para Colombia en toma de muestras y dopaje, Confederación Suramericana de Fútbol, CSF, y Federación Internacional de Fútbol Asociado, FIFA. Miembro de la Comisión Médica, Comité Olímpico Colombiano, COC. 


\section{Una conceptualización necesaria}

La reflexión inmediata que se suscita cuando se analiza con sentido profundo el tema del dopaje, oteando lo psicosocial y lo ético, requiere hacer precisiones teóricas para que el abordaje que es indispensable hacer no se quede ni en lo superficial del término dopaje -de hecho, una desviación negativa del deporte-, ni en lo filosófico, lo romántico, lo idealista o lo ético.

\section{La cultura}

Para introducir el tema, la cultura es un conjunto de rasgos distintivos, espirituales y materiales, intelectuales y afectivos que caracterizan una sociedad o a un grupo social en un periodo determinado. El término "cultura" engloba además modos de vida, ceremonias, arte, invenciones, tecnología, sistemas de valores, derechos fundamentales del ser humano, tradiciones y creencias. A través de la cultura se expresa el ser humano, toma conciencia de sí mismo, cuestiona sus realizaciones, busca nuevos significados y crea obras que trascienden. La cultura física, entre tanto, que se deriva del concepto general de cultura y del concepto particular de física, en su concepción más amplia, es el componente de la cultura universal que sintetiza las categorías, las legitimidades, las instituciones y los bienes materiales creados para la valoración del ejercicio físico con el fin de perfeccionar el potencial biológico y, de manera implícita, el potencial espiritual del ser humano. La cultura física estudia la relación del ser humano con su medio a través de la actividad física, la cual se proyecta en su cultura corporal y espiritual. Tiene un carácter físico educativo y se evidencia en formas de realización diferentes y muy variadas: la educación física (pedagogía del movimiento); el deporte (proceso teórico y táctico, asociado a planes metodológicos del entrenamiento); la recreación física (asociada a procesos lúdicos y culturales); y la rehabilitación física (que incorpora programas de reacondicionamiento físico para diferentes edades, con patologías o sin patologías, que se orientan a través de las evaluaciones funcionales).

\section{El deporte}

El deporte ha impulsado para sí y para su entorno un desarrollo constante, en el cual han participado todas las fuerzas productivas, todas las clases sociales, todos los regímenes políticos, actuando en forma dinámica e importante en la determinación de objetivos, formas y organizaciones, de modo que se sobreponga a la ex- plotación y la destrucción del ser humano y más bien fortalezca el bienestar y el perfeccionamiento de la sociedad.

El deporte ha convocado el interés de líderes de la política, la ciencia y el desarrollo, porque tiene la capacidad de producir el cambio de actitud de la comunidad, aspecto necesario para asegurar la paz, el desarrollo y la convivencia sostenibles, único camino posible para garantizar la proyección de la vida en el planeta Tierra.

La cultura y el deporte constituyen en su conjunto un fenómeno social que participa en el proceso de formación de la personalidad de los individuos, sobre la base de las condiciones de la vida material, en la cual se han desarrollado, según las relaciones de producción, fortaleciendo además la esperanza, los valores y las perspectivas en el devenir histórico del ser humano, debido a que contribuye en forma determinante a la formación multilateral y armónica del individuo desde los primeros años de vida hasta la adultez.

El deporte constituye hoy el tema de conversación más frecuente, más difundido por todos los medios de comunicación social, incluso entre personas que no se conocen y que poco conocen el tema deportivo. Es parte de la vida diaria. El estadio, la cancha, la pista o el escenario son por excelencia lugares que permiten en forma inmediata el acceso para la práctica respectiva, garantizándola; facilitan la cohesión social y la culturización. En distintos contextos el deporte y su práctica son muy antiguos; su carácter lúdico, que se establece a través de la competencia, y el ritual que se condensa en las reglas, le proporcionan el valor de aliviar tensiones sociales y reforzar el orden comunitario. El deporte es, por tanto, un fenómeno cultural sólido.

La tecnología ha influido en el avance y el desarrollo del deporte. No obstante, su predominio a cualquier costo, aun el de su propia existencia, sistematiza, mediatiza, sustituye y disfraza funciones (biológicas, mecánicas, sociales, económicas y políticas) del individuo, y pone en jaque al ser humano y su permanencia en este planeta.

El juego es libertad e invención, fantasía y disciplina al mismo tiempo. Sin embargo, los juegos de la sociedad se nutren de la realidad construida por los medios de comunicación. No son gratuitos, "condicionan", y su objetivo de fondo, al igual que lo hacen los medios masivos de comunicación, se presentan en forma velada: utilizan el humor, la habilidad, las peripecias entretenidas, el mercadeo, el liderazgo y la fascinación social, para "jugar" a la apariencia del "hacer creer". Se juegan con la alegría o la tristeza, la pobreza, la ilusión colecti- 
va, la guerra o el amor, y llegan incluso a manipular los sentimientos más profundos de quien juega o de quien ve jugar. La tecnología, además, promociona el consumismo; nos ametralla con la publicidad y juega con las ambiciones de un pueblo que tiene carencias, que tiene necesidades que en ocasiones son minimizadas por estos eventos. Es la que recluta la familia en un cuarto, frente a la caja de ilusiones, a la caja de catarsis, a la caja de sueños. Pero el deporte es más que esto. Es preservación de la salud, es desarrollo social, ético y moral, es desarrollo intelectual, es formación y educación integral.

\section{La vida y el derecho a la vida}

La vida se origina a partir de la fusión de dos células o dos gametos, que, para el caso de la especie humana, constituye todo un proceso (la fecundación) que combina no sólo el material genético sino probablemente un gran componente actitudinal y espiritual. La vida humana se desenvuelve durante todo el ciclo vital, lo cual permite su expresión ontológica y jurídica. Es atribuible al ser humano, lo cual se traduce en un derecho, que, por su misma naturaleza, hace que el ser humano sea el dueño único e insustituible de su propia vida, como lo es de su conciencia, de su conocimiento y de su razón.

Surge, entonces, el derecho a la vida, que presupone respeto y protección. Pues bien, ese derecho es atribuido al ser humano. Como el ser humano es el dueño de su propia vida, este derecho resulta natural, es decir, como dueño que es de su propio ser, como lo es de sus bienes, de sus saberes, y de sus poderes.

La protección y el respeto que se derivan del derecho a la vida son una responsabilidad específica e insoslayable del Estado, de la sociedad civil, de la Iglesia, de los progenitores, de todos aquellos que como agentes del entorno social intervienen en el proceso que gesta el verdadero respeto y la protección de la vida. Una visión de ello son los derechos humanos (Thomas Paine): "constituyen la conjunción de los derechos naturales que le corresponden al ser humano por el solo hecho de existir"; éstos son diferentes de los derechos civiles, que "son aquellos que le asisten al ser humano por el hecho de ser miembro de una sociedad".

Cuando el ser humano cumple todo su ciclo vital y se extingue en él la vida (biológica) y llega la muerte, se extinguen el derecho natural y su acción en la sociedad (derecho civil).

El derecho a la vida encarna la propiedad de universalidad (igualdad en tiempo y espacio), sin importar condición social ni calidad. Es irrenunciable, porque no se puede renunciar a la vida, al propio ser, porque ello sig- nifica renunciar a su integridad personal y a sus interrelaciones ontológicas y teleológicas. El derecho a la vida es inalienable, como lo reza la Declaración Universal de los Derechos Humanos (10 de diciembre de 1948): “La libertad, la justicia y la paz en el mundo tienen como base el reconocimiento de la dignidad intrínseca y de los derechos iguales e inalienables de todos los miembros de la familia humana".

La vida es el compendio de ser, pensar, hacer y tener, enmarcado en lo moral y lo ético; se fundamenta en la conciencia personal, que imprime su propia existencia de acuerdo con su propia dignidad. La vida y el derecho natural son la base fundamental del devenir histórico y social de la especie humana, que ha trascendido de generación en generación.

\section{La lógica y el pensamiento humano}

Dos conceptos clave llaman aquí la atención: la lógica, entendida como lo que puede aceptarse como la verdad o aquello que aparece en el pensamiento individual y que, a la luz de la estructura cognitiva de un ser particular, éste lo adopta como lógico u obvio.

El pensamiento permite al ser humano crear, razonar, analizar e interpretar integralmente el Cosmos; facilita el estudio riguroso y controvertido de la realidad; analiza los diferentes problemas que surgen de la contradicción y la contrastación dialéctica. Procura así encontrar la solución de problemas, lo que lo acerca a la verdad, interna o externa, del entorno social o psicoafectivo, y facilita el desarrollo creciente del pensamiento que nace de la percepción reflejada del contexto, que para este análisis es esa realidad social afectada por fenómenos políticos, económicos, psicológicos y culturales, que se interiorizan y se agudizan en sus resultados, cuando se afecta el estado biopsicosocial del ser humano, su calidad evolutiva y el desarrollo autónomo del pensamiento y de sus convicciones.

Todo lo que existe en la naturaleza tiene una racionalidad, una razón de ser. Las fuerzas cósmicas, los procesos bélicos, las crisis sociales, tienen su razón de ser descle el punto de vista de sus propios actores y de sus propias concepciones o verdades, y es a través de esta contradicción, de esta dinámica, de donde emerge lo racional para unos o lo irracional para otros.

Hasta aquí resulta evidente que el ser humano se mueva mediante modelos (prejuicios) o paradigmas que afectan su comportamiento en sus variadas dimensiones; que le dan orden y disciplina a ese comportamiento, y que en el caso del contexto social, la estructura de la razón y el contacto dinámico cognoscitivo, permiten 
el ser humano transforme sus experiencias, venza paradigmas para enrumbar su desarrollo; conocer la ciencia matemática, biológica o social, mediante la interrelación del sujeto consciente con el objeto (por conocer) a fin de que el primero se apropie progresivamente del conocimiento (propiedades) del objeto.

Ahora bien, la subjetividad del pensamiento significa el poder pensar por sí mismo sin que nadie pueda hacerlo, interferirlo o sugerirlo. Así, el ser humano es él mismo, con pensamiento autónomo y soberano, con dominio para entender y comprender con análisis crítico su entorno.

Ese hacer autónomo e independiente del pensamiento debe respetar la autonomía y la soberanía del pensamiento de los otros seres humanos afectivos, funcionales o sociales.

\section{La Libertad}

Se entiende como la facultad que tiene el hombre para elegir entre varias opciones, sin violencia externa, ni presión interna, y que en consecuencia lo hacen responsable de su conducta.

La libertad promueve la deliberación, el análisis, la dialéctica permanente del pensamiento; facilita la expresión de los derechos legítimos del hombre como prioridades incuestionables. La libertad le da la posibilidad de expresarse filosóficamente; de plantear postulados; de consensar y de negociar aspectos inherentes a su desarrollo integral.

\section{La conducta social}

La sociología por definición, hace referencia a los hechos sociales, y éste es su objeto de estudio. No sólo se debe conocer y entender la conducta humana, sino su comportamiento social.

La estructura social es un sistema o conjunto de elementos unidos, relacionados entre sí, que ocupan un lugar en ese sistema y se mantienen en equilibrio. Todos ocupan un puesto y desempeñan un rol o papel determinado en la sociedad, en función del estatus que establece el rol que cumplen en la sociedad. Cada estatus y cada rol deben tener deberes y derechos, los cuales se inician desde la gestación, durante la evolución y hasta la muerte.

Es precisamente cuando el hombre se humaniza, que defiende su valor específico en su realidad contextual, para que de su análisis genere cambios, y estas acciones tengan repercusión frente a un hecho social, de manera que pueda defenderse la vida como derecho natural, su rol en la sociedad como derecho social, el derecho de participar dialécticamente con liderazgo y credibilidad. Así que cuando se confronte con la realidad o con los fenómenos sociales, su opinión tenga validez, pueda apropiarse de la razón, del análisis, de la negociación y del consenso de conceptos y operaciones, que puedan ofrecer decisiones a fin de obtener el respeto entre los grupos, se fortalezcan los derechos, los deberes y la dignidad.

Las reflexiones anteriores ubican el análisis en el campo de las diferentes dimensiones que afectan al ser humano en su capacidad innata de expresarse a través del movimiento:

- La dimensión biológica, que establece características ligadas a su individualidad, a los procesos orgánicos y fisiológicos, en relación estrecha con su carga genética, única e irrepetible. Aún hoy se plantea la clonación.

- La dimensión psicoafectiva, que por sus características se relaciona con la práctica de actividad física y deporte, como elementos básicos de la motivación, el sacrificio y la proyección espiritual.

- La dimensión sociocultural, de la cual ya se han hecho algunos planteamientos.

En su conjunto, estas dimensiones van estructurando también el concepto moderno de salud, cuyo alcance no sólo es biopsicosocial, sino político-legal, cuya nota característica es la salud como derecho y obligación universal, que tiene reconocimiento legal y participación estatal y se basa en los derechos del ser humano. Las conductas de salud se encuentran vinculadas con el estilo de vida, que se define como el conjunto de pautas de conducta y hábitos cotidianos de una persona, entendida aquella en sus más amplias dimensiones, en el contexto de la cultura, con valores y costumbres, que por supuesto, se apoyan, entre otros, en conductas preventivas, que protegen las actividades cotidianas del ser humano, entre ellas el deporte, y que en su conjunto se encuentran firmemente establecidas y arraigadas en el repertorio de comportamiento de cada individuo.

\section{Más sobre lo social}

Desde el punto de vista sociológico (MEN, 1989: 87), las manifestaciones del deporte, sus actores, los procedimientos y la organización, por mínima que sea, constituyen un entramado tan complejo que bien puede afirmarse que éste configura una nueva institución social, con una relevancia similar a la de las instituciones ya clásicas de la familia, la religión, la educación y el trabajo. Sus perspectivas económicas, su impacto políti- 
co y la contribución que el deporte hace a la formación y la transmisión de valores democráticos constituyen, sin lugar a dudas, uno de los movimientos sociales más dinámicos de la contemporaneidad y de la globalización, con amplísimas repercusiones socioculturales.

Es claro que en su proceso histórico (MEN, 1989: 87) el deporte ha superado las manifestaciones íntimamente asociadas al rito religioso, la magia y la danza, desde los tiempos más remotos, que en su conjunto se encaminaban a aplacar la ira de los dioses o a regular los fenómenos naturales. En la medida en que evolucionan las relaciones de producción, y la sociedad va diferenciándose en grupos o clases más definidas, el deporte va adquiriendo características propias: se convierte en medio de distracción, de regulación de la agresividad y de competencia no violenta. Poco a poco aparece el deporte como un "hecho social" distinto y diferenciado de la religión. Así, avanza gestando su propia autonomía y sus manifestaciones culturales, como se evidencia en la Grecia clásica con la organización de la Olimpiada.

En la era moderna, la revolución industrial, el advenimiento del Capitalismo, la globalización más recientemente, han determinado un cambio cualitativo en los contenidos sociales del deporte. La práctica del deporte comienza a ser sometida a la lógica de las relaciones de producción capitalista y a convertirse en una actividad motivada por el afán de lucro, que requiere la trama compleja de los medios de publicidad y de grandes inversiones en equipos y escenarios, y en la cual los espectadores son un mercado que es necesario conquistar mediante técnicas comerciales; el deportista es un simple instrumento de producción, y el dirigente es una empresa que debe planificar la producción y producir utilidades. Surge así el deporte "espectáculo", el deporte que genera capital y utilidades, lo cual desvirtúa la lúdica, consustancial al ser humano. A partir de este momento, se desvanece su papel sublimador de la agresividad, para convertirse en un espacio donde se exacerban de modo artificial los conflictos, las contradicciones de la sociedad, la presencia creciente del "dolo", camino expedito para escalar posiciones sociales, sin importar cómo lograrlo.

En la actualidad puede decirse que coexisten de manera dialéctica dos grandes tendencias del deporte: una asociada a la racionalidad capitalista, que impulsa la comercialización y el espectáculo, que ya ha alcanzado los gimnasios, el modelaje, las escuelas deportivas y otras manifestaciones, y otra que promueve los valores vinculados al perfeccionamiento físico, psicológico y social, la salud y su calidad, a la competencia sana, a la afirmación de hábitos de convivencia y solidaridad ciudadanos, y a la satisfacción de una necesidad fundamental de las sociedades contemporáneas, que es la de la recreación, el tiempo libre y la deportividad.

En el documento "Carta internacional de la educación y el deporte" de la Unesco (1978), y en la "Declaración de Moscú” (1988 - II Conferencia Internacional de Ministros y altos funcionarios encargados de la educación física y el deporte), se considera el deporte como "portador de los más nobles valores humanistas" y "un medio poderoso de acercar a los pueblos, de favorecer el entendimiento internacional y de servir a la causa de la paz".

Se condenan "ciertos fenómenos contra los valores educativos, culturales y morales del deporte, que pueden empañar su imagen, como el dopaje, la comercialización excesiva, la violencia, el nacionalismo y el profesionalismo".

Derivado de estos documentos, se reivindica el derecho que tiene todo ser humano de acceder a la educación física y al deporte, porque éstos constituyen instrumentos insustituibles de la lucha contra las plagas sociales, como el alcoholismo, el consumo de drogas, la marginación, y la pobreza económica, afectiva y psicosocial.

De un 20 a un $25 \%$ de la población del país está integrada por personas jóvenes (14 a 25 años), y un 20\% aproximadamente de niños y niñas de cualquier estrato social desean o realizan actividad física o deporte popular, en un contexto en el cual se destacan la falta de oportunidad de empleo remunerativo (estudios realizados por la Universidad Nacional de Colombia, la Contraloría y asesores de la OEA, plantean de 17 a 23\% de pobreza en el país); el debilitamiento de la capacidad socializadora de la familia y la escuela; y la falta de participación política, que gestan la búsqueda de otras alternativas sociales y políticas al margen de la legalidad. El deporte puede ser la tabla de salvación, en el medio de las presiones comerciales o de las esperanzas de un futuro mejor.

Es claro para el deporte que la mayoría de niños, niñas y adolescentes y futuros talentos de la actividad deportiva emergen de las comunidades más populares, pero que, por lo mismo, carecen de los servicios más elementales o condiciones básicas inherentes al desarrollo psicosocial y cultural de cualquier comunidad contemporánea. La problemática propia de estos sectores se enmarca en la marginación, la pobreza, la falta de calidad del empleo, el subempleo o el mismo desempleo, que afectan, sin lugar a dudas, el estado de salud 
ambiental y cultural de estas comunidades, lo cual impide asegurar procesos adecuados de crecimiento, desarrollo y madurez psicomotriz de los futuros semilleros de talentos deportivos.

La participación comunitaria, que es ante todo una práctica social a través de la cual muy diversos actores sociales, portadores de intereses y fuerzas diferenciales (Misión: Bogotá siglo XXI,1990: 64-157), establecen sus relaciones y construyen una dinámica social conflictiva, que en continuo movimiento define los consensos y los disensos, construye y modifica la sociedad. Allí, en ese entorno, se mueven los jóvenes y los futuros talentos. Allí se entrecruzan sus ambiciones, sus ilusiones, sus deseos, que sentados en el mundo hiperdinámico del deporte, se proyectan con la ansiedad e incluso la temeridad de lograr un éxito deportivo, a cualquier costo o en cualquier circunstancia o con cualquier método, si con él se alivian frustraciones económicas y sociales y se consolida, así sea fugazmente, "un liderazgo" que penetre su entorno social y familiar. $Y$ en este entorno se encuentran progenitores, familia, maestros y entrenadores, que esperanzados en sus "pupilos", comulgan también con estrategias ajenas al entrenamiento cabal y persistente, o al concepto insoslayable de la individualidad biológica, para ofrecer, estimular o enmascarar el camino o método más impropio o poco ético de alcanzar la cima del éxito, con menoscabo total de sus dirigidos.

Es incuestionable que el índice global de calidad de vida (índice mixto compuesto por vivienda, educación, salud, transporte, ingresos, trabajo, espacio público y seguridad ciudadana) se presenta bajo o muy bajo en estas comunidades $y$, por tanto, su efecto se presenta con mucha evidencia sobre las poblaciones más expuestas: niñas, niños y jóvenes.

Es realmente impactante y determinante no sólo el efecto que la calidad de vida y las necesidades básicas más sentidas tienen en el comportamiento, el carácter y la personalidad de estos grupos, sino también en sus propias convicciones y reflexiones frente a la vida, su. futuro y el deporte.

Acosta (2003) $)^{1}$, en un documento de trabajo sobre la localidad de Santa Fe, desvela asuntos críticos e indicadores que reflejan la problemática social de las

\footnotetext{
Documento de trabajo. La Universidad Incca toma como referentes dos documentos elaborados por la Secretaría de Salud del Distrito Capital: el primero titulado Localidad de Santa Fe, aún sin publicar, y una monografía sobre la localidad de Santa Fe, en versión preliminar para la revisión por parte del Departamento Administrativo de Planeación Distrital, en su página web de 2003.
}

comunidades donde se entrecruzan variables económicas, educativas, de salud, de desarrollo cultural, que afectan directamente la niñez y la juventud y donde se aprecia que si algo hace cohesión social es el deporte. Si algo puede ofrecer al joven algún sendero esperanzador, es el deporte; en medio de limitaciones y frustraciones, confrontándose con la dura realidad de una oportunidad, rodeado de toda suerte de defectos de la sociedad que detenta los medios de producción, o del mensajero que le señala el camino... pero que le exige resultados y lo explota o lo conmina a utilizar los métodos o las prácticas más lesivas a su integralidad y a su conducta. En suma, es innegable la contribución de lo social a la problemática del dopaje.

\section{El juego limpio}

El "juego limpio" significa mucho más que el simple respeto de las reglas: abarca los conceptos de amistad, respeto al adversario y espíritu deportivo. Más que un comportamiento, es un modo de pensar. El concepto se extiende a la lucha contra las trampas, contra el arte de engañar sin vulnerar las reglas, contra el dopaje, la violencia física y verbal, la desigualdad de oportunidades, la comercialización excesiva y la corrupción.

El "juego limpio" es un concepto positivo, siempre que se practique con lealtad. El código de ética considera el deporte como una actividad sociocultural que enriquece la sociedad y la amistad entre las naciones. El deporte es considerado, así mismo, como una actividad que permite a la persona conocerse, expresarse y realizarse mejor; desarrollarse, adquirir conocimientos prácticos y demostrar sus capacidades; el deporte hace posible la interacción social, es fuente de disfrute y aporta bienestar y salud. El deporte, con su extensa red de clubes y de aficionados, ofrece la ocasión de participar y de asumir responsabilidades sociales. Además, la participación responsable en determinadas actividades puede coadyuvar al desarrollo de la sensibilidad respecto al medio ambiente.

El código de ética reconoce que la participación de niños, niñas y adolescentes en las actividades deportivas se inserta en un entorno social más amplio. Admite que el individuo y la sociedad sólo pueden aprovechar plenamente las ventajas potenciales del deporte si el "juego limpio" deja de ser un concepto secundario para convertirse en un principio central. Reconoce que todas las personas que, en una forma directa o indirecta, favorecen e influyen en la experiencia que niños, niñas $y$ adolescentes viven en el deporte, deben conceder una prioridad absoluta a dicho concepto. 


\section{La ética en el deporte}

El deporte es una actividad sociocultural que permite el enriquecimiento del individuo en el seno de la sociedad y que potencia la amistad entre los pueblos, el intercambio entre las regiones y las naciones y, en suma, el conocimiento y la relación entre las personas.

El deporte contribuye a mejorar la relación, el conocimiento y la expresión personales. Es un factor de integración social, fuente de disfrute, salud y bienestar. La realización de estos valores permite la participación de la sociedad desde unas pautas distintas de las que a menudo constituyen las actitudes sociales más tradicionales. Estas pautas de participación y relación social deben contribuir al desarrollo de determinadas sensibilidades, como la del respeto a las distintas nacionalidades y razas, al medio ambiente y a la calidad de vida como factor de convivencia social. La preservación de estos valores hace necesario que el deporte recupere algunos de sus elementos tradicionales y que, por tanto, el respeto a las reglas del juego, la lealtad, la ética y la deportividad sean elementos de vertebración de los participantes en el mismo.

La ética es esencial tanto en la actividad como en la gestión deportiva. Dicho comportamiento permite encauzar la rivalidad y la controversia deportivas desde unas pautas diferentes y aceptables socialmente que puedan producir ejemplaridad frente a otras formas de relación social.

\section{Deportividad}

La ética impone una actuación decidida contra la trampa, la manipulación y la adulteración de cualquier índole, de los resultados y las actuaciones deportivas. Especialmente, este compromiso alcanza la lucha contra el dopaje, la violencia física y verbal, la segregación por razones de raza, origen o pensamiento y la corrupción que pueda ser debida a los fuertes intereses comerciales que rodean al mundo del deporte de nuestros días. La deportividad es ante todo un principio positivo. La sociedad se enriquece con la práctica deportiva y con lo que ésta supone de fomento de los valores más elevados de la personalidad a la vez que con el intercambio personal y social que el mismo supone. El deporte ayuda a conocerse mejor, a expresarse y a desarrollarse en un entorno social en el cual se valore la salud y el bienestar.

El código de ética reconoce que todas las entidades deportivas o personas que, en forma directa o indirecta, estén relacionadas con la actividad deportiva, deben beneficiarse de las ventajas morales y culturales del deporte si la deportividad y la ejemplaridad son la preocupación principal de los dirigentes deportivos y de cuantas entidades y asociaciones tengan relación con el deporte.

Las personas, y específicamente, deportistas, padres, educadores, técnicos, árbitros, directivos, administradores y médicos, así como los deportistas de alta competición que sirven de modelo, deberán guardar un comportamiento de respeto y de compromiso con la deportividad. El código ético debe aplicarse a todas las personas, con independencia de que participen como voluntarios o en calidad de profesionales. También quienes asistan a una competición deportiva, en su condición de espectadores, deberán asumir su cuota de responsabilidad y de buen comportamiento.

\section{Lo psicológico}

El impacto de la práctica del dopaje en el área psicológica de los deportistas es realmente crítico, con afectación en su conducta, en sus hábitos, en el proceder en relación con sus congéneres o con su equipo de trabajo humano y técnico, incluso con alteraciones en la capacidad que cada atleta debe tener para realizar técnicas de autoobservación y desempeñar de manera apropiada el rol que implica la observación precisa de los métodos de entrenamiento, orientados a mejorar su ámbito de actuación, consolidar su autoconfianza, y fortalecer su propio imaginario: pensamientos, creencias, sensaciones, etcétera.

El componente psicológico está firmemente ligado a procesos relacionados con la conducta de los atletas, el ambiente deportivo, su comportamiento en equipo, el ocio, la salud en su concepción más amplia, la consecución de objetivos, el mantenimiento de habilidades, y en general hace parte de la formación integral del deportista: por tanto, prácticas contrarias a todo este acervo, entre ellas el dopaje, afectan profundamente los componentes psicoafectivos, conductuales, sus estados emocionales y la visión que en relación con su contexto de actuación social, familiar y deportivo, desarrollen en el ámbito de sus diferentes desempeños. Alcanzan percepciones equivocadas, se desenvuelven en la ansiedad y el estrés, limitan la comunicación adecuada con su equipo o con su entrenador, generan intolerancia, frustración e inseguridad que llevan a los deportistas a resultados deformables en lo deportivo y que afectan su componente biopsicosocial, predisponiéndolo a trastornos psicológicos, psiquiátricos y, en un gran porcentaje, a fenómenos de adicción y toxicomanía. A muchos deportistas 


\section{REFLEXIONES ORIGINALES}

estos trastornos los marginan definitivamente de la vida en sociedad y los condenan a ser personas ausentes al desarrollo humano, y ajenos a la familia y a la sociedad, con implicaciones muy serias y graves en todos sus contextos de actuación.

Realizado el análisis anterior, que aún requiere mayor profundidad, se hace necesario aproximar algunas conclusiones:

- El dopaje es un ejemplo de los numerosos intentos que en el transcurso de la historia el ser humano ha realizado para mejorar de modo artificial su propia resistencia a la fatiga, a la participación en la guerra, en tareas cotidianas y laborales de exigencia, a la actividad física y al deporte, mezclando para ello frecuentemente esta práctica con la magia y la brujería.

- La organización económica del mundo contemporáneo, la globalización y el impacto financiero impulsan a multitud de personas de diferentes disciplinas deportivas a buscar incrementar en forma artificial su rendimiento físico e incluso psíquico, más allá de su límite natural, sin tener en cuenta el riesgo intrínseco que conlleva esta actitud. Se busca con ilusión desbordada, y con poco fundamento científico, el producto milagroso que sea capaz de transformar a la persona común y corriente en un superhombre o una supermujer.

- En 1956, C. Faroux escribió en el periódico L'Equipe:

se trata de una plaga que debe combatirse por todas las fuerzas vivas de la nación. ¿Quién será el primero que se atreverá a atacar este mal que ya ha causado tantos estragos? La llamada a la conciencia individual, respecto de este tema, puede ser un móvil poderoso...

- El deporte y el deportista que lo practica quieren un deporte limpio y sano, en consonancia con sus ideales y con su dignidad. El verdadero deportista es el que nunca se dopa, el que entrena con constancia,

\section{Referencias}

Cadavid Villa, Rubén A. Ensayo sobre los caminos para la paz. Bogotá: Universidad Incca de Colombia, Revista Institucional, número extraordinario.

(2004). Estado del arte histórico-filosófico de la categoría movimiento como functor [conector o enlace] entre la filosofía y la cultura física y su influencia en el desarrollo integral del sacrificio, y con la ilusión de alcanzar un gran rendimiento, dentro de sus capacidades y sus condiciones biológicas y técnicas.

La práctica del dopaje es contraria a la ética y a las lealtades deportivas; destruye los beneficios que de modo tradicional se han buscado; afecta el desarrollo integral del deportista, menoscaba el derecho a su libertad y su dignidad, porque el dopaje envilece los valores más preciados del deporte y el deportista, y los convierte en objeto, al cual se utiliza y se manipula. El deportista es un instrumento mediante el cual se persiguen objetivos poco altruistas. Es una máquina que hay que amortizar en breve plazo y que debe rendir al máximo. El dopaje contradice la finalidad prioritaria del deporte, que es propender a una mejor salud física, mental y social. Disminuye su carácter lúdico. Al tratar de mejorar artificialmente cualidades y potencialidades, contraviene el principio esencial del deporte de alto rendimiento, según el cual éste debe ser justo y equitativo. Por tanto, introduce el engaño y la mentira, y como conducta dolosa destruye la confianza que resulta necesaria en una actividad social que ante todo debe ser auténtica.

Es inadmisible que el liderazgo y el ejemplo deportivos devengan de una práctica que atente contra los preceptos sagrados de la deportividad, el juego limpio, la competencia franca y sana.

La construcción de un mundo mejor, más pacífico, más auténtico, que proteja principalmente a la juventud, se logrará si unidos todos los estamentos de la sociedad $y$, por supuesto, del deporte en su conjunto, luchamos para detener el tráfico y el consumo de las drogas de uso ilícito, porque el deporte debe estar al servicio del dèsarrollo armonioso de la humanidad, estimulando su práctica contra la toxicomanía; movilizando para ello recursos humanos y financieros y creando programas de promoción y prevención que estimulen su práctica sana, haciendo alarde de la ética y de los valores que hacen del ser humano su verdadera razón de ser.

hombre contemporáneo. Bogotá: Universidad Incca de Colombia, Facultad de Ciencias Humanas y Sociales.

. (1999). Fundamentos filosóficos, políticos y socio-

históricos de las teorías sociales y pedagógicas. Ensayo. Bogotá: Universidad Incca de Colombia.

Castaño A., César A. (1999). El derecho a la vida. Una visión desde la filosofía. Bogotá: Universidad Incca de Colombia, Maestría en Filosofía. 
Confederación Deportiva Mexicana. (1996). ¿Qué... con el doping? Ciudad de México: Confederación Deportiva Mexicana. Artículo en Internet.

Ministerio de Educación Nacional-Coldeportes. (1989). Plan indicativo del deporte en Colombia. El Deporte: un nuevo derecho social. Bogotá: MEN-Coldeportes.

Misión: Bogotá siglo XXI. (1990). El futuro de la capital. Un estudio de prospectiva urbana, descentralización y participación urbana. Santa Fe de Bogotá: mimeografía.
Unesco. Revista ATP. Comunicaciones científicas mexicanas. S.A. de C.V. Unesco.

Unesco. (1998, octubre). Declaración Mundial sobre Educación Superior en el siglo XXI: visión y acción. París: Unesco.

www.rusport.es/leg-esp/listadoping 99.htm www.mecon.ar/recursos/econoti/revista 1/pag.3 htm www.deusto.es/publicaciones/pableusk/pag.15 www.droydepo.htm 